\title{
MULTIPLE POSITIVE SOLUTIONS FOR THE NONLINEAR SCHRÖDINGER-POISSON SYSTEM
}

\author{
Weiming Liu* and Miaomiao Niu \\ Hubei Normal University, School of Mathematics and Statistics \\ Huangshi, 435002, P. R. China; whu.027@163.com \\ Beijing Normal University, School of Mathematical Sciences \\ Beijing, 100875, P. R. China; miaomiaoniu@mail.bnu.edu.cn
}

Abstract. We consider the following Schrödinger-Poisson system in $\mathbf{R}^{3}$

$$
\begin{cases}-\Delta u+u+\alpha K(|x|) \Phi(x) u=|u|^{p-2} u, & x \in \mathbf{R}^{3}, \\ -\Delta \Phi=K(|x|) u^{2}, & x \in \mathbf{R}^{3},\end{cases}
$$

where $2<p<6, \alpha$ can be regarded as a parameter and $K(r)(r=|x|)$ is a positive continuous function. There are constants $a \in \mathbf{R}$ and $b \in\left(0, \frac{1}{2}\right]$, such that $K(r) \sim r^{a} e^{-b r}$, as $r \rightarrow+\infty$. Then, $(0.1)$ possesses a non-radial positive solution with exactly m maximum points for suitable range of $\alpha$.

\section{Introduction and main result}

The following Schrödinger-Poisson system

$$
\begin{cases}-\Delta u+V(x) u+K(x) \Phi(x) u=|u|^{p-2} u, & x \in \mathbf{R}^{3}, \\ -\Delta \Phi=K(x) u^{2}, & x \in \mathbf{R}^{3},\end{cases}
$$

has been studied extensively by many researchers, where $2<p<6$. This system has been first introduced in [5] as a physical model describing a charged wave interacting with its own electrostatic field in quantum mechanic. The unknowns $u$ and $\Phi$ represent the wave functions associated to the particle and electric potential, and functions $V$ and $K$ are respectively an external potential and nonnegative density charge. We refer to [5] and the references therein for more physical background.

In recent years, there has been increasing interest in studying problem (1.1). In the case of $V(x) \equiv 1, K(x) \equiv \lambda>0$, the existence of radially symmetric positive solutions of system (1.1) was obtained by D'Aprile and Mugnai in [9] and Ruiz in [20] for $p \in(3,6)$. Azzollini and Pomponio in [4] established the existence of ground state solutions for $p \in(3,6)$. For $p \in(2,3), \lambda=1$, Ruiz in [20] proved that (1.1) does not admit any nontrivial solution. When $K(x) \equiv 1$ and $V(x)$ is not a constant, the authors proved that there exist radially symmetric solutions concentrate on the spheres in $[12,14]$ and a positive bound state solution concentrates on the local minimum of the potential $V$ in [13]. By using constrained minimization on the signchanging Nehari manifold and the Brouwer degree theory, Wang and Zhou in [23]

https://doi.org/10.5186/aasfm.2017.4218

2010 Mathematics Subject Classification: Primary 35J10, 35J20, 35J60.

Key words: Schrödinger-Poisson system, non-radial positive solutions, variational methods, Lyapunov-Schmidt reduction.

Acknowledgements: The authors thank the referee and the editor for helpful discussions and suggestions. This project is supported by the National Natural Science Foundation of China (Grant No. 11601139).

*Corresponding author. 
studied the existence of sign-changing solutions for (1.1). Ruiz and Vaira in [21] constructed the multi-bump solutions whose bumps concentrated around the local minimum of the potential $V$. The proofs explored in [21] are based on a singular perturbation, essentially a Lyapunov-Schmidt reduction method. In [11], He and Li concerned with the problem (1.1) with critical nonlinearity. For the more general nonlinearity $f(u)$, by applying the method of penalized functions, Sun et al. in [22] proved the system (1.1) has one nontrivial solution in the case $3<p \leq 4$. For more related results, one can refer to $[3,1,5,7,10,15,24,25]$ and the references therein.

Our motivation to study (1.1) mainly comes from the results: In [18], Lin et al. proved the single Schrödinger equation

$$
-\Delta u+(1+\epsilon K(x)) u=|u|^{p-2} u, \quad x \in \mathbf{R}^{N},
$$

has multi-bump solutions with the condition:

$$
K(x) \in C\left(\mathbf{R}^{N}, \mathbf{R}^{+}\right), \quad \lim _{|x| \rightarrow \infty} K(x)=0, \quad \lim _{|x| \rightarrow \infty} \frac{\ln (K(x))}{|x|}=0 .
$$

In [17], Li et al. consider the infinitely many positive solutions of the following Schrödinger-Poisson system

$$
\begin{cases}-\Delta u+u+K(|x|) \Phi(x) u=Q(|x|)|u|^{p-2} u, & x \in \mathbf{R}^{3}, \\ -\Delta \Phi=K(|x|) u^{2}, & x \in \mathbf{R}^{3},\end{cases}
$$

where $K(|x|)$ and $Q(|x|)$ are bounded and positive functions, $2<p<6 . K(|x|)$ and $Q(|x|)$ have the following expansions:

$$
\begin{aligned}
& K(r)=\frac{a}{r^{m}}+O\left(\frac{1}{r^{m+\theta}}\right), \quad\left(a>0, m>\frac{1}{2}, \theta>0\right), \quad r=|x| \rightarrow+\infty \\
& Q(r)=Q_{0}+\frac{b}{r^{n}}+O\left(\frac{1}{r^{n+\vartheta}}\right),\left(Q_{0}>0, b \in \mathbf{R}, n>1, \vartheta>0\right), r=|x| \rightarrow+\infty
\end{aligned}
$$

As we can see, the expansion (1.4) is a special form of (1.2). Motivated by all results mentioned above, it is very natural for us to pose an interesting question: if the condition $\lim _{|x| \rightarrow \infty} \frac{\ln (K(x))}{|x|}<0$ holds, can we obtain the multiple positive solutions for the problem (1.1)? Especially, in [19], Long and Peng studied the existence of multiple positive solutions for the single Schrödinger equation under the above codition. Now, our answer is positive. We consider the following condition $(K)$ which satisfies $\lim _{|x| \rightarrow \infty} \frac{\ln (K(x))}{|x|}<0$.

In this paper, we studied the following Schrödinger-Poisson system

$$
\begin{cases}-\Delta u+u+\alpha K(|x|) \Phi(x) u=|u|^{p-2} u, & x \in \mathbf{R}^{3}, \\ -\Delta \Phi=K(|x|) u^{2}, & x \in \mathbf{R}^{3},\end{cases}
$$

where $K(r)(r=|x|)$ is a positive continuous function, $2<p<6$. We assume that $K(r)$ satisfies the following condition:

$(K)$ There are constants $a \in \mathbf{R}$ and $b \in\left(0, \frac{1}{2}\right]$, such that

$$
K(r) \sim r^{a} e^{-b r}
$$

as $r \rightarrow+\infty$.

We summarize our main results as follows.

Theorem 1.1. If $K(r)$ satisfies $(K)$, for a fixed integer $m>1$, provided one of the following conditions holds: 
(1) If $b>\sin \frac{\pi}{m}$, then $\alpha>\alpha_{1}$ for suitable large $\alpha_{1}>0$;

(2) If $b<\sin \frac{\pi}{m}$, then $0<\alpha<\alpha_{2}$ for suitable small $\alpha_{2}>0$, where $\alpha_{1}$ and $\alpha_{2}$ are only dependent on $b$ and $m$.

Then problem (1.6) possesses a non-radial positive solution with exactly m maximum points.

In the following, we sketch the main idea in the proof of Theorem 1.1. The Sobolev space $H^{1}\left(\mathbf{R}^{3}\right)$ is endowed with the standard norm

$$
\|u\|=\sqrt{\langle u, u\rangle}, \quad u \in H^{1}\left(\mathbf{R}^{3}\right),
$$

which is induced by the inner product

$$
\left\langle v_{1}, v_{2}\right\rangle=\int_{\mathbf{R}^{3}} \nabla v_{1} \nabla v_{2}+\int_{\mathbf{R}^{3}} v_{1} v_{2}
$$

The homogeneous Sobolev space

$$
D^{1,2}\left(\mathbf{R}^{3}\right)=\left\{u \in L^{2^{*}}\left(\mathbf{R}^{3}\right): \nabla u \in L^{2}\left(\mathbf{R}^{3}\right)\right\},
$$

with the norm

$$
\|u\|_{D^{1,2}}=\left(\int_{\mathbf{R}^{3}}|\nabla u|^{2}\right)^{\frac{1}{2}} .
$$

For every $u \in H^{1}\left(\mathbf{R}^{3}\right)$, the Riesz theorem implies that there exists a unique $\Phi_{u} \in D^{1,2}\left(\mathbf{R}^{3}\right)$ such that $-\Delta \Phi_{u}=K(x) u^{2}$ and $\Phi_{u}$ can be represented by

$$
\Phi_{u}(x)=\frac{1}{4 \pi} \int_{\mathbf{R}^{3}} \frac{K(y) u^{2}(y)}{|x-y|} d y .
$$

Furthermore, one has

$$
\left\|\Phi_{u}\right\|_{D^{1,2}} \leq c\|u\|^{2}
$$

where $c>0$. Thus system (1.6) can be reduced into equation

$$
-\Delta u+u+K(|x|) \Phi_{u} u=|u|^{p-2} u \text {. }
$$

The solution of (1.6) can be approximated by using the solution $U$ of the following problem

$$
\left\{\begin{array}{l}
-\Delta u+u=u^{p-1}, \quad u>0 \text { in } \mathbf{R}^{3}, \\
u(0)=\max _{x \in \mathbf{R}^{3}} u(x) .
\end{array}\right.
$$

It is well-known that the unique solution $U$ of (1.9) satisfies $U(x)=U(|x|)$ and $U^{\prime}<0$ (see [16]).

For any positive integer $m>1$, let us define

$$
y_{j}=\left(r \cos \frac{2(j-1) \pi}{m}, r \sin \frac{2(j-1) \pi}{m}, 0\right):=\left(y_{j}^{\prime}, 0\right), \quad j=1,2, \ldots, m,
$$

where $r \in\left[\frac{(1-\tau) \ln \alpha}{b-\sin \frac{\pi}{m}}, \frac{(1+\tau) \ln \alpha}{b-\sin \frac{\pi}{m}}\right]$ for some $\tau>0$ small enough. Define

$$
\begin{aligned}
H=\{u: u & \in H^{1}\left(\mathbf{R}^{3}\right), u \text { is even in } x_{h}, h=2,3 ; u\left(r \cos \theta, r \sin \theta, x_{3}\right) \\
& \left.=u\left(r \cos \left(\theta+\frac{2 \pi j}{m}\right), r \sin \left(\theta+\frac{2 \pi j}{m}\right), x_{3}\right), j=1,2, \ldots, m\right\} .
\end{aligned}
$$

Let

$$
U_{\mathbf{y}}(x)=\sum_{j=1}^{m} U_{y_{j}}(x)
$$


where $U_{y_{j}}(\cdot)=U\left(\cdot-y_{j}\right)$.

Theorem 1.1 is a direct consequence of the following result.

Theorem 1.2. Suppose $K(r)$ satisfies $(K), b \neq \sin \frac{\pi}{m}$ and provided one of following conditions holds:

(1) If $b>\sin \frac{\pi}{m}$, then $\alpha>\alpha_{1}$ for suitable large $\alpha_{1}>0$;

(2) If $b<\sin \frac{\pi}{m}$, then $0<\alpha<\alpha_{2}$ for suitable small $\alpha_{2}>0$, where $\alpha_{1}$ and $\alpha_{2}$ are only dependent on $b$ and $m$.

Then (1.6) has a positive solution $u_{m}$ of the form

$$
u_{m}=U_{\mathbf{y}_{m}}(x)+w_{m},
$$

where $w_{m} \in H, r_{m} \in\left[\frac{(1-\tau) \ln \alpha}{b-\sin \frac{\pi}{m}}, \frac{(1+\tau) \ln \alpha}{b-\sin \frac{\pi}{m}}\right], \tau>0$ small enough and as $\alpha \rightarrow+\infty\left(\right.$ or $\left.0^{+}\right)$, $\left\|w_{m}\right\|_{H^{1}} \rightarrow 0$.

Our paper is organized as follows. In section 2, we will carry out LyapunovSchmidt reduction. Then, we will study the reduced finite-dimensional problem and prove our mian result in section 3. Some technical estimates are left in the appendix.

\section{Finite-dimensional reduction}

We begin the Lyapunov-Schmidt for the proof of Theorem 1.2. Assume

$$
r \in \Lambda_{m}:=\left[\frac{(1-\tau) \ln \alpha}{b-\sin \frac{\pi}{m}}, \frac{(1+\tau) \ln \alpha}{b-\sin \frac{\pi}{m}}\right],
$$

where $\tau>0$ small enough. Define

$$
I(u)=\frac{1}{2} \int_{\mathbf{R}^{3}}|\nabla u|^{2}+\frac{1}{2} \int_{\mathbf{R}^{3}} u^{2}+\frac{\alpha}{4} \int_{\mathbf{R}^{3}} K(|x|) \Phi_{u} u^{2}-\frac{1}{p} \int_{\mathbf{R}^{3}}|u|^{p}, \quad \forall u \in H .
$$

It is easy to check that

$$
\begin{aligned}
& \int_{\mathbf{R}^{3}} \nabla u_{1} \nabla u_{2}+\int_{\mathbf{R}^{3}} u_{1} u_{2}-(p-1) \int_{\mathbf{R}^{3}} U_{\mathbf{y}}^{p-2} u_{1} u_{2}+\alpha \int_{\mathbf{R}^{3}} K(|x|) \Phi_{U_{\mathbf{y}}} u_{1} u_{2} \\
& +2 \alpha \int_{\mathbf{R}^{3}} K(|x|)\left(\int_{\mathbf{R}^{3}} \frac{K(|y|)}{|x-y|} U_{\mathbf{y}} u_{1} d y\right) U_{\mathbf{y}} u_{2}, \quad u_{1} u_{2} \in H,
\end{aligned}
$$

is a bounded bi-linear functional in $H$. Hence, by Lax-Milgram Theorem there is a bounded linear operator $\mathcal{L}$ from $H$ to $H$, such that

$$
\begin{aligned}
\left\langle\mathcal{L} u_{1}, u_{2}\right\rangle= & \int_{\mathbf{R}^{3}} \nabla u_{1} \nabla u_{2}+\int_{\mathbf{R}^{3}} u_{1} u_{2}-(p-1) \int_{\mathbf{R}^{3}} U_{\mathbf{y}}^{p-2} u_{1} u_{2}+\alpha \int_{\mathbf{R}^{3}} K(|x|) \Phi_{U_{\mathbf{y}}} u_{1} u_{2} \\
& +2 \alpha \int_{\mathbf{R}^{3}} K(|x|)\left(\int_{\mathbf{R}^{3}} \frac{K(|y|)}{|x-y|} U_{\mathbf{y}} u_{1} d y\right) U_{\mathbf{y}} u_{2}, \quad u_{1} u_{2} \in H .
\end{aligned}
$$

The following result implies that $\mathcal{L}$ is invertible in $H$.

Lemma 2.1. There are positive constants $C$, small $\alpha_{2}$ and $\alpha_{1}$ sufficiently large such that for $\alpha>\alpha_{1}$ when $b>\sin \frac{\pi}{m}$ or $0<\alpha<\alpha_{2}$ when $b<\sin \frac{\pi}{m}$,

$$
\|\mathcal{L} u\| \geq C\|u\|, \quad u \in H .
$$

Proof. We only prove the lemma for the case $b>\sin \frac{\pi}{m}$. Here we prove it by a contradiction argument. Suppose to the contrary that there exist $\alpha_{k} \rightarrow+\infty$ and $u_{k} \in H$ with

$$
\left\|\mathcal{L} u_{k}\right\|=o(1)\left\|u_{k}\right\| .
$$


Then we have

$$
\left\langle\mathcal{L} u_{k}, \varphi\right\rangle=o(1)\left\|u_{k}\right\|\|\varphi\|, \forall \varphi \in H
$$

We may assume that $\left\|u_{k}\right\|^{2}=1$.

Denote

$$
\Omega_{j}=\left\{x=\left(x^{\prime}, x_{3}\right) \in \mathbf{R}^{2} \times \mathbf{R}:\left\langle\frac{x^{\prime}}{\left|x^{\prime}\right|}, \frac{y_{j}^{\prime}}{\left|y_{j}^{\prime}\right|}\right\rangle \geq \cos \frac{\pi}{m}\right\}, \quad j=1,2, \cdots, m .
$$

By symmetry, we see from (2.2),

$$
\begin{aligned}
& \int_{\Omega_{1}} \nabla u_{k} \nabla \varphi+\int_{\Omega_{1}} u_{k} \varphi-(p-1) \int_{\Omega_{1}} U_{\mathbf{y}}^{p-2} u_{k} \varphi+\alpha \int_{\Omega_{1}} K(|x|) \Phi_{U_{\mathbf{y}}} u_{k} \varphi \\
& +2 \alpha \int_{\Omega_{1}} K(|x|)\left(\int_{\mathbf{R}^{3}} \frac{K(|y|)}{|x-y|} U_{\mathbf{y}} u_{k} d y\right) U_{\mathbf{y}} \varphi=o(1)\|\varphi\|, \quad \varphi \in H .
\end{aligned}
$$

Particularly, choosing $\varphi=u_{k}$ we get

$$
\begin{aligned}
& \int_{\Omega_{1}}\left|\nabla u_{k}\right|^{2}+\int_{\Omega_{1}} u_{k}^{2}-(p-1) \int_{\Omega_{1}} U_{\mathbf{y}}^{p-2} u_{k}^{2}+\alpha \int_{\Omega_{1}} K(|x|) \Phi_{U_{\mathbf{y}}} u_{k}^{2} \\
& +2 \alpha \int_{\Omega_{1}} K(|x|)\left(\int_{\mathbf{R}^{3}} \frac{K(|y|)}{|x-y|} U_{\mathbf{y}} u_{k} d y\right) U_{\mathbf{y}} u_{k}=o(1)
\end{aligned}
$$

and

$$
\int_{\mathbf{R}^{3}}\left|\nabla u_{k}\right|^{2}+\int_{\mathbf{R}^{3}} u_{k}^{2}=1
$$

Let $\tilde{u}_{k}(x)=u_{k}\left(x-y_{1}\right)$. It is easy to check that for any $R>0$, we can choose $k$ large enough such that $B_{R}\left(y_{1}\right) \subset \Omega_{1}$. Consequently, (2.5) yields that

$$
\int_{B_{R}(0)}\left|\nabla \tilde{u}_{k}\right|^{2}+\int_{B_{R}(0)} \tilde{u}_{k}^{2} \leq 1 .
$$

Thus we may assume that there exists a $u \in H^{1}\left(\mathbf{R}^{3}\right)$ such that as $k \rightarrow+\infty$,

$$
\tilde{u}_{k} \rightarrow u \text {, weakly in } H^{1}\left(\mathbf{R}^{3}\right)
$$

and

$$
\tilde{u}_{k} \rightarrow u \text {, strongly in } L_{\text {loc }}^{2}\left(\mathbf{R}^{3}\right) .
$$

Noting that $\tilde{u}_{k}$ is even in $x_{h}, h=2,3$, then $u$ is even in $x_{h}, h=2,3$. On the other hand, from

$$
\int_{\mathbf{R}^{3}} \frac{\partial U_{y_{1}}}{\partial r} U_{y_{1}}^{p-2} u_{k}=0
$$

we obtain

So $u$ satisfies

$$
\int_{\mathbf{R}^{3}} \frac{\partial U}{\partial x_{1}} U^{p-2} \tilde{u}_{k}=0 .
$$

$$
\int_{\mathbf{R}^{3}} \frac{\partial U}{\partial x_{1}} U^{p-2} u=0 .
$$

Now we prove that $u$ satisfies

$$
-\Delta u+u-(p-1) U^{p-2} u=0, \text { in } \mathbf{R}^{3} .
$$

Define

$$
\tilde{H}=\left\{\varphi: \varphi \in H^{1}\left(\mathbf{R}^{3}\right), \int_{\mathbf{R}^{3}} \frac{\partial U}{\partial x_{1}} U^{p-2} \varphi=0\right\} .
$$


For any $R>0$, let $\varphi \in C_{0}^{\infty}\left(B_{R}(0)\right) \cap \tilde{H}$ be any function, satisfying that $\varphi$ is even in $x_{h}, h=2,3$. Then $\varphi_{1}(x)=: \varphi\left(x-y_{1}\right) \in C_{0}^{\infty}\left(B_{R}\left(y_{1}\right)\right)$. By using (2.4), we find

$$
\int_{\mathbf{R}^{3}} \nabla u \nabla \varphi+\int_{\mathbf{R}^{3}} u \varphi-(p-1) \int_{\mathbf{R}^{3}} U^{p-2} u \varphi=0 .
$$

Furthermore, since $u$ is even in $x_{h}, h=2,3,(2.7)$ is true for any function $\varphi \in$ $C_{0}^{\infty}\left(\mathbf{R}^{3}\right)$, which is odd in $x_{h}, h=2,3$. Therefore, (2.7) is true for any $\varphi \in$ $C_{0}^{\infty}\left(B_{R}(0)\right) \cap \tilde{H}$. Since $C_{0}^{\infty}\left(\mathbf{R}^{3}\right)$ is dense in $H^{1}\left(\mathbf{R}^{3}\right)$, it is easy to prove that

$$
\int_{\mathbf{R}^{3}} \nabla u \nabla \varphi+\int_{\mathbf{R}^{3}} u \varphi-(p-1) \int_{\mathbf{R}^{3}} U^{p-2} u \varphi=0, \quad \forall \varphi \in \tilde{H} .
$$

But (2.8) is true for $\varphi=\frac{\partial U}{\partial x_{1}}$. Thus (2.8) is true for any $\varphi \in H^{1}\left(\mathbf{R}^{3}\right)$, and hence $u=c \frac{\partial U}{\partial x_{1}}$ because $u$ is even in $x_{h}, h=2,3$. By (2.6), we find $u=0$. Consequently,

$$
\int_{B_{R}\left(y_{1}\right)} u_{k}^{2}=o(1), \quad \forall R>0 .
$$

By the Hölder inequality and Lemma A.2, we have

$$
\begin{aligned}
& \left|\alpha \int_{\Omega_{1}} K(|x|) \Phi_{U_{\mathbf{y}}} u_{k}^{2}+2 \alpha \int_{\Omega_{1}} K(|x|)\left(\int_{\mathbf{R}^{3}} \frac{K(|y|)}{|x-y|} U_{\mathbf{y}} u_{k} d y\right) U_{\mathbf{y}} u_{k}\right| \\
& \leq \alpha r^{a} e^{-b r} C \int_{\mathbf{R}^{3}} u_{k}^{2}+\alpha \int_{\mathbf{R}^{3}} K(|x|)\left|\Phi_{U_{\mathbf{y}}}\right|^{\frac{1}{2}}\left|\Phi_{u_{k}}\right|^{\frac{1}{2}} U_{\mathbf{y}} u_{k} \\
& \leq \alpha r^{a} e^{-b r} C+\alpha r^{a} e^{-b r} r^{\frac{a}{2}} e^{-\frac{b}{2} r} C \int_{\mathbf{R}^{3}}\left|\Phi_{u_{k}}\right|^{\frac{1}{2}} U_{\mathbf{y}} u_{k} \\
& \leq \alpha r^{a} e^{-b r} C+\alpha r^{a} e^{-b r} C\left\|u_{k}\right\|^{2}\left(\int_{\mathbf{R}^{3}} U_{\mathbf{y}}^{2}\right)^{\frac{1}{2}} \rightarrow 0 .
\end{aligned}
$$

Thus, by (2.2) and $(K)$, we have

$$
\begin{aligned}
o(1)= & \int_{\Omega_{1}}\left|\nabla u_{k}\right|^{2}+\int_{\Omega_{1}} u_{k}^{2}-(p-1) \int_{\Omega_{1}} U_{\mathbf{y}}^{p-2} u_{k}^{2}+\alpha \int_{\Omega_{1}} K(|x|) \Phi_{U_{\mathbf{y}}} u_{k}^{2} \\
& +2 \alpha \int_{\Omega_{1}} K(|x|)\left(\int_{\mathbf{R}^{3}} \frac{K(|y|)}{|x-y|} U_{\mathbf{y}} u_{k} d y\right) U_{\mathbf{y}} u_{k} \\
\geq & \int_{\Omega_{1}}\left|\nabla u_{k}\right|^{2}+\int_{\Omega_{1}} u_{k}^{2}+o(1)+o_{R}(1) \int_{\Omega_{1}} u_{k}^{2} \geq \frac{1}{2}>0,
\end{aligned}
$$

which is impossible for large $R$.

Now, we prove the following proposition.

Proposition 2.2. Under the assumptions of Lemma 2.1, there exists a $C^{1}$ map with respect to $r$ from $\Lambda_{m}$ to $H: \varphi=\varphi(r)$, satisfying $\varphi \in H$, and

$$
\left\langle\frac{\partial J(\varphi)}{\partial \varphi}, v\right\rangle=0, \quad \forall v \in H
$$

Moreover, there is a small $\tau>0$, such that

$$
\|\varphi\| \leq C\left(\alpha e^{-(1-\tau) 2 b r}+e^{-\min \{p-1-\sigma, 2-\sigma\} r \sin \frac{\pi}{m}}\right) .
$$

Proof. Denote

$$
J(\varphi)=I\left(U_{\mathbf{y}}+\varphi\right), \quad \varphi \in H .
$$


By direct computation, we have

$$
\begin{aligned}
J(\varphi)= & I\left(U_{\mathbf{y}}+\varphi\right)=\frac{1}{2} \int_{\mathbf{R}^{3}}\left|\nabla U_{\mathbf{y}}+\nabla \varphi\right|^{2}+\frac{1}{2} \int_{\mathbf{R}^{3}}\left(U_{\mathbf{y}}+\varphi\right)^{2} \\
& +\frac{\alpha}{4} \int_{\mathbf{R}^{3}} K(|x|) \Phi_{U_{\mathbf{y}}+\varphi}\left(U_{\mathbf{y}}+\varphi\right)^{2}-\frac{1}{p} \int_{\mathbf{R}^{3}}\left|U_{\mathbf{y}}+\varphi\right|^{p} \\
= & \frac{1}{2} \int_{\mathbf{R}^{3}}\left|\nabla U_{\mathbf{y}}\right|^{2}+\frac{1}{2} \int_{\mathbf{R}^{3}} U_{\mathbf{y}}^{2}+\frac{\alpha}{4} \int_{\mathbf{R}^{3}} K(|x|) \Phi_{U_{\mathbf{y}}} U_{\mathbf{y}}^{2}-\frac{1}{p} \int_{\mathbf{R}^{3}}\left|U_{\mathbf{y}}\right|^{p} \\
& +\int_{\mathbf{R}^{3}}\left(\sum_{j=1}^{m} U_{y_{j}}^{p-1}-U_{\mathbf{y}}^{p-1}\right) \varphi+\alpha \int_{\mathbf{R}^{3}} K(|x|) \Phi_{U_{\mathbf{y}}} U_{\mathbf{y}} \varphi \\
& +\frac{1}{2} \int_{\mathbf{R}^{3}}|\nabla \varphi|^{2}+\frac{1}{2} \int_{\mathbf{R}^{3}} \varphi^{2}-\frac{p-1}{2} \int_{\mathbf{R}^{3}}\left|U_{\mathbf{y}}\right|^{p-2} \varphi^{2}+\frac{\alpha}{2} \int_{\mathbf{R}^{3}} K(|x|) \Phi_{U_{\mathbf{y}}} \varphi^{2} \\
& +\alpha \int_{\mathbf{R}^{3}} K(|x|)\left(\int_{\mathbf{R}^{3}} \frac{K(|y|)}{|x-y|} U_{\mathbf{y}} \varphi d y\right) U_{\mathbf{y}} \varphi \\
& +\alpha \int_{\mathbf{R}^{3}} K(|x|) \Phi_{\varphi} U_{\mathbf{y}} \varphi+\frac{\alpha}{4} \int_{\mathbf{R}^{3}} K(|x|) \Phi_{\varphi} \varphi^{2}-\frac{1}{p} \int_{\mathbf{R}^{3}}\left|U_{\mathbf{y}}+\varphi\right|^{p}+\frac{1}{p} \int_{\mathbf{R}^{3}}\left|U_{\mathbf{y}}\right|^{p} \\
& +\int_{\mathbf{R}^{3}}\left|U_{\mathbf{y}}\right|^{p-1} \varphi+\frac{p-1}{2} \int_{\mathbf{R}^{3}}\left|U_{\mathbf{y}}\right|^{p-2} \varphi^{2} .
\end{aligned}
$$

Hence,

$$
J(\varphi)=J(0)+f(\varphi)+\frac{1}{2}\langle\mathcal{L} \varphi, \varphi\rangle+R(\varphi)
$$

where

$$
f(\varphi)=\int_{\mathbf{R}^{3}}\left(\sum_{j=1}^{m} U_{y_{j}}^{p-1}-U_{\mathbf{y}}^{p-1}\right) \varphi+\alpha \int_{\mathbf{R}^{3}} K(|x|) \Phi_{U_{\mathbf{y}}} U_{\mathbf{y}} \varphi .
$$

$\mathcal{L}$ is the bounded linear map from $H$ to $H$ in Lemma 2.1, and

$$
\begin{aligned}
R(\varphi)= & \alpha \int_{\mathbf{R}^{3}} K(|x|) \Phi_{\varphi} U_{\mathbf{y}} \varphi+\frac{\alpha}{4} \int_{\mathbf{R}^{3}} K(|x|) \Phi_{\varphi} \varphi^{2}-\frac{1}{p} \int_{\mathbf{R}^{3}}\left|U_{\mathbf{y}}+\varphi\right|^{p}+\frac{1}{p} \int_{\mathbf{R}^{3}}\left|U_{\mathbf{y}}\right|^{p} \\
& +\int_{\mathbf{R}^{3}}\left|U_{\mathbf{y}}\right|^{p-1} \varphi+\frac{p-1}{2} \int_{\mathbf{R}^{3}}\left|U_{\mathbf{y}}\right|^{p-2} \varphi^{2} .
\end{aligned}
$$

It is not difficult to verify that $f(\varphi)$ is a bounded linear functional in $H$, so there exists an $f_{m} \in H$ such that

$$
f(\varphi)=\left\langle f_{m}, \varphi\right\rangle .
$$

Thus, to find a critical point for $J(\varphi)$, we only need to solve

$$
f_{m}+\mathcal{L} \varphi+R^{\prime}(\varphi)=0 .
$$

From Lemma 2.1 we know that $\mathcal{L}$ is invertible. Therefore, (2.11) can be rewritten as

$$
\varphi=\mathcal{A}(\varphi)=:-\mathcal{L}^{-1} f_{m}-\mathcal{L}^{-1} R^{\prime}(\varphi) .
$$

Set

$$
\mathcal{N}=\left\{\varphi: \varphi \in H,\|\varphi\| \leq \alpha e^{-\left(1-\tau-\tau_{1}\right) 2 b r}+e^{-\min \left\{p-1-\sigma-\tau_{1}, 2-\sigma-\tau_{1}\right\} r \sin \frac{\pi}{m}}\right\} .
$$

where $\tau_{1}>0$ sufficiently small. When $2<p \leq 3$, we can verify that

$$
\left\|R^{\prime}(\varphi)\right\| \leq C\|\varphi\|^{p-1} .
$$


Hence Lemma 2.3 below implies

$$
\begin{aligned}
\|\mathcal{A}(\varphi)\| \leq & C\left\|f_{m}\right\|+C\|\varphi\|^{p-1} \\
\leq & C \alpha e^{-(1-\tau) 2 b r}+C e^{-\min \{p-1-\sigma, 2-\sigma\} r \sin \frac{\pi}{m}}+C\left(\alpha e^{-\left(1-\tau-\tau_{1}\right) 2 b r}\right. \\
& \left.+e^{-\min \left\{p-1-\sigma-\tau_{1}, 2-\sigma-\tau_{1}\right\} r \sin \frac{\pi}{m}}\right)^{p-1} \\
\leq & \alpha e^{-\left(1-\tau-\tau_{1}\right) 2 b r}+e^{-\min \left\{p-1-\sigma-\tau_{1}, 2-\sigma-\tau_{1}\right\} r \sin \frac{\pi}{m}} .
\end{aligned}
$$

Thus, $\mathcal{A}$ maps $\mathcal{N}$ into $\mathcal{N}$ when $2<p \leq 3$.

Meanwhile, when $2<p \leq 3$, we see

$$
\left\|R^{\prime \prime}(\varphi)\right\| \leq C\|\varphi\|^{p-2} .
$$

Thus,

$$
\begin{aligned}
& \left\|\mathcal{A}\left(\varphi_{1}\right)-\mathcal{A}\left(\varphi_{2}\right)\right\|=\left\|\mathcal{L}^{-1} R^{\prime}\left(\varphi_{1}\right)-\mathcal{L}^{-1} R^{\prime}\left(\varphi_{2}\right)\right\| \\
& \leq C\left\|R^{\prime}\left(\varphi_{1}\right)-R^{\prime}\left(\varphi_{2}\right)\right\| \leq C\left\|R^{\prime \prime}\left(\varepsilon \varphi_{1}+(1-\varepsilon) \varphi_{2}\right)\right\|\left\|\varphi_{1}-\varphi_{2}\right\| \\
& \leq C\left(\left\|\varphi_{1}\right\|^{p-2}+\left\|\varphi_{2}\right\|^{p-2}\right)\left\|\varphi_{1}-\varphi_{2}\right\| \leq \frac{1}{2}\left\|\varphi_{1}-\varphi_{2}\right\|,
\end{aligned}
$$

where $\varepsilon \in(0,1)$. Thus, we have proved that when $2<p \leq 3, \mathcal{A}$ is a contraction map.

When $p>3$, by the Hölder inequality and the Sobolev inequality, we get

$$
\begin{aligned}
\left|\left\langle R^{\prime}(\varphi), \xi\right\rangle\right| & \mid 2 \alpha \int_{\mathbf{R}^{3}} K(|x|)\left(\int_{\mathbf{R}^{3}} \frac{K(|y|)}{|x-y|} \varphi \xi d y\right) U_{\mathbf{y}} \varphi+\alpha \int_{\mathbf{R}^{3}} K(|x|) \Phi_{\varphi} U_{\mathbf{y}} \xi+\alpha \int_{\mathbf{R}^{3}} K(|x|) \Phi_{\varphi} \varphi \xi \\
& -\int_{\mathbf{R}^{3}}\left|U_{\mathbf{y}}+\varphi\right|^{p-1} \xi+\int_{\mathbf{R}^{3}}\left|U_{\mathbf{y}}\right|^{p-1} \xi+(p-1) \int_{\mathbf{R}^{3}}\left|U_{\mathbf{y}}\right|^{p-2} \varphi \xi \mid \\
\leq & \left|2 \alpha \int_{\mathbf{R}^{3}} K(|x|)\left(\int_{\mathbf{R}^{3}} \frac{K(|y|)}{|x-y|} \varphi \xi d y\right) U_{\mathbf{y}} \varphi+\alpha \int_{\mathbf{R}^{3}} K(|x|) \Phi_{\varphi} U_{\mathbf{y}} \xi+\alpha \int_{\mathbf{R}^{3}} K(|x|) \Phi_{\varphi} \varphi \xi\right| \\
& +\left|\int_{\mathbf{R}^{3}}\right| U_{\mathbf{y}}+\left.\varphi\right|^{p-1} \xi-\int_{\mathbf{R}^{3}}\left|U_{\mathbf{y}}\right|^{p-1} \xi-(p-1) \int_{\mathbf{R}^{3}}\left|U_{\mathbf{y}}\right|^{p-2} \varphi \xi \mid \\
\leq & C \alpha r^{a} e^{-b r} \int_{\mathbf{R}^{3}}\left|\Phi_{\varphi}\right|^{\frac{1}{2}}\left|\Phi_{\xi}\right|^{\frac{1}{2}} U_{\mathbf{y}}|\varphi|+C \alpha r^{a} e^{-b r}\left(\int_{\mathbf{R}^{3}}\left|\Phi_{\varphi}\right|^{6}\right)^{\frac{1}{6}}\left(\int_{\mathbf{R}^{3}}|\xi|^{\frac{12}{5}}\right)^{\frac{5}{12}}\left(\int_{\mathbf{R}^{3}}\left|U_{\mathbf{y}}\right|^{\frac{12}{5}}\right)^{\frac{5}{12}} \\
& +C \alpha r^{a} e^{-b r}\left(\int_{\mathbf{R}^{3}}\left|\Phi_{\varphi}\right|^{6}\right)^{\frac{1}{6}}\left(\int_{\mathbf{R}^{3}}|\xi|^{\frac{12}{5}}\right)^{\frac{5}{12}}\left(\int_{\mathbf{R}^{3}}|\varphi|^{\frac{12}{5}}\right)^{\frac{5}{12}}+C \int_{\mathbf{R}^{3}}\left|U_{\mathbf{y}}\right|^{p-3}|\varphi|^{2}|\xi| \\
\leq & C \alpha^{\frac{b \tau-\sin \frac{\pi}{m}}{m}}\|\varphi\|^{2}\|\xi\|+C \alpha^{\frac{b \tau-\sin \frac{\pi}{m}}{\frac{m}{m}}}\left\|\Phi_{\varphi}\right\|_{D^{1,2}}\|\xi\|+C \alpha^{\frac{b \tau-\sin \frac{\pi}{m}}{\frac{m}{m}}}\left\|\Phi_{\varphi}\right\|_{D^{1,2}}\|\xi\|\|\varphi\| \\
& +C\left(\int_{\mathbf{R}^{3}}\left(\left|U_{\mathbf{y}}\right|^{p-3}|\varphi|^{2}\right)^{\frac{p}{p-1}}\right)^{\frac{p-1}{p}}\|\xi\| \\
\leq & C\|\varphi\|^{2}\|\xi\|+C\|\varphi\|^{2}\|\xi\|+C\|\varphi\|^{3}\|\xi\|+C\left(\int_{\mathbf{R}^{3}}|\varphi|^{\frac{2 p}{p-1}}\right)^{\frac{p-1}{p}}\|\xi\| .
\end{aligned}
$$

Hence, we deduce that

$$
\left\|R^{\prime}(\varphi)\right\| \leq C\left(\|\varphi\|^{2}+\|\varphi\|^{3}\right) .
$$


For the estimate of $\left\|R^{\prime \prime}(\varphi)\right\|$, we have

$$
\begin{aligned}
& \left|R^{\prime \prime}(\varphi)(\xi, \eta)\right| \\
& =\mid 2 \alpha \int_{\mathbf{R}^{3}} K(|x|)\left(\int_{\mathbf{R}^{3}} \frac{K(|y|)}{|x-y|} \eta \xi d y\right) U_{\mathbf{y}} \varphi+2 \alpha \int_{\mathbf{R}^{3}} K(|x|)\left(\int_{\mathbf{R}^{3}} \frac{K(|y|)}{|x-y|} \varphi \xi d y\right) U_{\mathbf{y}} \eta \\
& \quad+2 \alpha \int_{\mathbf{R}^{3}} K(|x|)\left(\int_{\mathbf{R}^{3}} \frac{K(|y|)}{|x-y|} \varphi \eta d y\right) U_{\mathbf{y}} \xi+2 \alpha \int_{\mathbf{R}^{3}} K(|x|)\left(\int_{\mathbf{R}^{3}} \frac{K(|y|)}{|x-y|} \varphi \xi d y\right) \varphi \eta \\
& \quad+\alpha \int_{\mathbf{R}^{3}} K(|x|) \Phi_{\varphi} \xi \eta-(p-1) \int_{\mathbf{R}^{3}}\left(U_{\mathbf{y}}+\varphi\right)^{p-2} \xi \eta+(p-1) \int_{\mathbf{R}^{3}} U_{\mathbf{y}}^{p-2} \xi \eta \mid \\
& \leq C\left(\|\varphi\|+\|\varphi\|^{2}\right)\|\xi\|\|\eta\|,
\end{aligned}
$$

which implies

$$
\left\|R^{\prime \prime}(\varphi)\right\| \leq C\left(\|\varphi\|+\|\varphi\|^{2}\right) .
$$

Thus, we have

$$
\begin{aligned}
\|\mathcal{A}(\varphi)\| \leq & C\left\|f_{m}\right\|+C\|\varphi\|^{2} \\
\leq & C \alpha e^{-(1-\tau) 2 b r}+C e^{-\min \{p-1-\sigma, 2-\sigma\} r \sin \frac{\pi}{m}} \\
& +C\left(\alpha e^{-\left(1-\tau-\tau_{1}\right) 2 b r}+e^{-\min \left\{p-1-\sigma-\tau_{1}, 2-\sigma-\tau_{1}\right\} r \sin \frac{\pi}{m}}\right)^{2} \\
\leq & \alpha e^{-\left(1-\tau-\tau_{1}\right) 2 b r}+e^{-\min \left\{p-1-\sigma-\tau_{1}, 2-\sigma-\tau_{1}\right\} r \sin \frac{\pi}{m}}
\end{aligned}
$$

and

$$
\begin{aligned}
& \left\|\mathcal{A}\left(\varphi_{1}\right)-\mathcal{A}\left(\varphi_{2}\right)\right\|=\left\|\mathcal{L}^{-1} R^{\prime}\left(\varphi_{1}\right)-\mathcal{L}^{-1} R^{\prime}\left(\varphi_{2}\right)\right\| \\
& \leq C\left\|R^{\prime}\left(\varphi_{1}\right)-R^{\prime}\left(\varphi_{2}\right)\right\| \leq C\left\|R^{\prime \prime}\left(\varepsilon \varphi_{1}+(1-\varepsilon) \varphi_{2}\right)\right\|\left\|\varphi_{1}-\varphi_{2}\right\| \leq \frac{1}{2}\left\|\varphi_{1}-\varphi_{2}\right\|,
\end{aligned}
$$

where $\varepsilon \in(0,1)$. Hence, $\mathcal{A}$ is also a contraction map from $\mathcal{N}$ to $\mathcal{N}$.

By the contraction mapping theorem, we see that there is a unique $\varphi$ such that (2.11) holds. Moreover, it follows from (2.12) and (2.13) that (2.9) holds.

Lemma 2.3. There exists a constant $C>0$, such that

$$
\left\|f_{m}\right\| \leq C\left(\alpha e^{-(1-\tau) 2 b r}+e^{-\min \{p-1-\sigma, 2-\sigma\} r \sin \frac{\pi}{m}}\right),
$$

where $\tau>0$ is an arbitrary small constant.

Proof. We recall

$$
f(\varphi)=\int_{\mathbf{R}^{3}}\left(\sum_{j=1}^{m} U_{y_{j}}^{p-1}-U_{\mathbf{y}}^{p-1}\right) \varphi+\alpha \int_{\mathbf{R}^{3}} K(|x|) \Phi_{U_{\mathbf{y}}} U_{\mathbf{y}} \varphi .
$$

Since it follows from (3.18) and (3.19) in [8], by Lemma A.1, we have

$$
\begin{aligned}
\left|\int_{\mathbf{R}^{3}} U_{\mathbf{y}}^{p-1} \varphi-\sum_{j=1}^{m} \int_{\mathbf{R}^{3}} U_{y_{j}}^{p-1} \varphi\right| & \leq \begin{cases}C \sum_{k \neq j} \int_{\mathbf{R}^{3}} U_{y_{k}}^{\frac{p-1}{2}} U_{y_{j}}^{\frac{p-1}{2}}|\varphi|, & \text { if } 2<p \leq 3, \\
C \sum_{k \neq j} \int_{\mathbf{R}^{3}} U_{y_{k}}^{p-2} U_{y_{j}}|\varphi|, \quad \text { if } p>3, & \leq C e^{-\min \{p-1-\tau, 2-\tau\} r \sin \frac{\pi}{m}}\|\varphi\|,\end{cases}
\end{aligned}
$$

where $\tau>0$ small enough. 
By the Hölder inequality, we have

$$
\begin{aligned}
& \left|\int_{\mathbf{R}^{3}} K(|x|) \Phi_{U_{\mathbf{y}}} U_{\mathbf{y}} \varphi\right|=\left|\sum_{j=1}^{m} \int_{\mathbf{R}^{3}} K(|x|) \Phi_{U_{\mathbf{y}}} U_{y_{j}} \varphi\right| \\
& \leq \sum_{j=1}^{m}\left(\int_{\mathbf{R}^{3}}\left(K(|x|) \Phi_{U_{\mathbf{y}}} U_{y_{j}}\right)^{2}\right)^{\frac{1}{2}}\left(\int_{\mathbf{R}^{3}} \varphi^{2}\right)^{\frac{1}{2}} \\
& \leq\left\|\Phi_{U_{\mathbf{y}}}\right\|_{D^{1,2}} \sum_{j=1}^{m}\left(\int_{\mathbf{R}^{3}}\left(K(|x|) U_{y_{j}}\right)^{3}\right)^{\frac{1}{3}}\|\varphi\| .
\end{aligned}
$$

Let us evaluate $\left\|\Phi_{U_{\mathbf{y}}}\right\|_{D^{1,2}}$ and $\int_{\mathbf{R}^{3}}\left(K(|x|) U_{y_{j}}\right)^{3}$.

$$
\begin{aligned}
\left\|\Phi_{U_{\mathbf{y}}}\right\|_{D^{1,2}}^{2}= & \int_{\mathbf{R}^{3}}\left|\nabla \Phi_{U_{\mathbf{y}}}\right|^{2}=\int_{\mathbf{R}^{3}} K(|x|) \Phi_{U_{\mathbf{y}}} U_{\mathbf{y}}^{2} \\
= & \sum_{j=1}^{m} \int_{\mathbf{R}^{3}} K(|x|) \Phi_{U_{\mathbf{y}}} U_{y_{j}}^{2}+\sum_{i \neq j} \int_{\mathbf{R}^{3}} K(|x|) \Phi_{U_{\mathbf{y}}} U_{y_{i}} U_{y_{j}} \\
\leq & C\left\|\Phi_{U_{\mathbf{y}}}\right\|_{D^{1,2}} \sum_{j=1}^{m}\left(\int_{\mathbf{R}^{3}}\left(K(|x|) U_{y_{j}}^{2}\right)^{\frac{6}{5}}\right)^{\frac{5}{6}} \\
& +C\left\|\Phi_{U_{\mathbf{y}}}\right\|_{D^{1,2}} \sum_{i \neq j}\left(\int_{\mathbf{R}^{3}}\left(K(|x|) U_{y_{i}} U_{y_{j}}\right)^{\frac{6}{5}}\right)^{\frac{5}{6}}
\end{aligned}
$$

and

$$
\begin{aligned}
& \int_{\mathbf{R}^{3}}\left(K(|x|) U_{y_{j}}\right)^{3}=\int_{\mathbf{R}^{3}}\left(K\left(\left|x+y_{j}\right|\right) U\right)^{3} \\
& =\int_{B_{(1-\kappa) r}(0)}\left(K\left(\left|x+y_{j}\right|\right) U\right)^{3}+\int_{\mathbf{R}^{3} \backslash B_{(1-\kappa) r}(0)}\left(K\left(\left|x+y_{j}\right|\right) U\right)^{3} \\
& \leq \int_{B_{(1-\kappa) r}(0)}\left|x+y_{j}\right|^{3 a} e^{-3 b\left|x+y_{j}\right|} U^{3}+\int_{\mathbf{R}^{3} \backslash B_{(1-\kappa) r}(0)} K^{3}\left(\left|x+y_{j}\right|\right) e^{-3(1-\kappa) r} \\
& =O\left(r^{3 a} e^{-3 b r}\right)+O\left(e^{-3(1-\kappa) r}\right) .
\end{aligned}
$$

Similarly,

$$
\int_{\mathbf{R}^{3}}\left(K(|x|) U_{y_{j}}^{2}\right)^{\frac{6}{5}}=O\left(r^{\frac{6}{5} a} e^{-\frac{6}{5} b r}\right)+O\left(e^{-\frac{12}{5}(1-\kappa) r}\right) .
$$

Denote

$$
\Omega_{j}=\left\{x=\left(x^{\prime}, x^{\prime \prime}\right) \in \mathbf{R}^{2} \times \mathbf{R}:\left\langle\frac{x^{\prime}}{\left|x^{\prime}\right|}, \frac{y_{j}^{\prime}}{\left|y_{j}^{\prime}\right|}\right\rangle \geq \cos \frac{\pi}{m}\right\}, j=1,2, \ldots, m .
$$

For any $x \in \Omega_{j}$, we have

$$
\left|x-y_{i}\right| \geq\left|x-y_{j}\right|, \quad \forall x \in \Omega_{j}, \quad i \neq j,
$$

then

$$
\left|x-y_{i}\right| \geq \frac{1}{2}\left|y_{i}-y_{j}\right|, \quad \forall x \in \Omega_{j}, \quad i \neq j .
$$

So, for all arbitrary constant $\eta \in(0,1)$, we find

$$
\begin{aligned}
U_{y_{i}} & \leq C e^{-\eta\left|x-y_{i}\right|} e^{-(1-\eta)\left|x-y_{i}\right|} \leq C e^{-\frac{\eta}{2}\left|y_{i}-y_{j}\right|} e^{-(1-\eta)\left|x-y_{j}\right|} \\
& \leq C e^{-\eta r \sin \frac{\pi}{m}} e^{-(1-\eta)\left|x-y_{j}\right|}, \quad \forall x \in \Omega_{j}, i \neq j .
\end{aligned}
$$


Using (2.19), we can obtain

$$
\begin{aligned}
& \int_{\mathbf{R}^{3}}\left(K(|x|) U_{y_{i}} U_{y_{j}}\right)^{\frac{6}{5}} \leq C m \int_{\Omega_{j}}\left(K(|x|) U_{y_{i}} U_{y_{j}}\right)^{\frac{6}{5}} \\
& \leq C m \int_{\Omega_{j}}\left(K(|x|) e^{-\eta r \sin \frac{\pi}{m}} e^{-(1-\eta)\left|x-y_{j}\right|} U_{y_{j}}\right)^{\frac{6}{5}} \\
& \leq C m e^{-\frac{6}{5} \eta r \sin \frac{\pi}{m}} \int_{\mathbf{R}^{3}}\left(K\left(\left|x+y_{j}\right|\right) e^{-(1-\eta)|x|} U\right)^{\frac{6}{5}} \\
&=C m e^{-\frac{6}{5} \eta r \sin \frac{\pi}{m}} \int_{B_{(1-\kappa) r}(0)}\left(K\left(\left|x+y_{j}\right|\right) e^{-(1-\eta)|x|} U\right)^{\frac{6}{5}} \\
&+C m e^{-\frac{6}{5} \eta r \sin \frac{\pi}{m}} \int_{\mathbf{R}^{3} \backslash B_{(1-\kappa) r}(0)}\left(K\left(\left|x+y_{j}\right|\right) e^{-(1-\eta)|x|} U\right)^{\frac{6}{5}} \\
& \leq C m e^{-\frac{6}{5} \eta r \sin \frac{\pi}{m}} \int_{B_{(1-\kappa) r}(0)}\left(\left|x+y_{j}\right|^{a} e^{-b\left|x+y_{j}\right|} e^{-(1-\eta)|x|} U\right)^{\frac{6}{5}} \\
& \quad+C m e^{-\frac{6}{5} \eta r \sin \frac{\pi}{m}} \int_{\mathbf{R}^{3} \backslash B_{(1-\kappa) r}(0)}\left(K\left(\left|x+y_{j}\right|\right) e^{-(2-\eta)|x|}\right)^{\frac{6}{5}} \\
& \leq C m e^{-\frac{6}{5} \eta r \sin \frac{\pi}{m}} \int_{\mathbf{R}^{3}}\left(\left|x+y_{j}\right|^{a} e^{-b\left|x+y_{j}\right|} e^{-(1-\eta)|x|} U\right)^{\frac{6}{5}} \\
& \quad+C m e^{-\frac{6}{5} \eta r \sin \frac{\pi}{m}} e^{-\frac{6}{5}(2-\eta)(1-\kappa) r} \int_{\mathbf{R}^{3}}\left(K\left(\left|x+y_{j}\right|\right)\right)^{\frac{6}{5}} \\
& \leq C m e^{-\frac{6}{5} \eta r \sin \frac{\pi}{m}}\left(r^{\frac{6}{5} a} e^{-\frac{6}{5} b r}+e^{-\frac{6}{5}(2-\eta)(1-\kappa) r}\right) .
\end{aligned}
$$

Inserting (2.16)-(2.20) into (2.15), we have

$$
\begin{aligned}
& \left|\int_{\mathbf{R}^{3}} K(|x|) \Phi_{U_{\mathbf{y}}} U_{\mathbf{y}} \varphi\right| \leq\left\|\Phi_{U_{\mathbf{y}}}\right\|_{D^{1,2}} \sum_{j=1}^{m}\left(\int_{\mathbf{R}^{3}}\left(K(|x|) U_{y_{j}}\right)^{3}\right)^{\frac{1}{3}}\|\varphi\| \\
& \leq C\left(\sum_{j=1}^{m}\left(\int_{\mathbf{R}^{3}}\left(K(|x|) U_{y_{j}}^{2}\right)^{\frac{6}{5}}\right)^{\frac{5}{6}}\right. \\
& \quad+\sum_{i \neq j}\left(\int_{\mathbf{R}^{3}}\left(K(|x|) U_{y_{i}} U_{y_{j}}\right)^{\frac{6}{5}}\right)^{\frac{5}{6}} \sum_{j=1}^{m}\left(\int_{\mathbf{R}^{3}}\left(K(|x|) U_{y_{j}}\right)^{3}\right)^{\frac{1}{3}}\|\varphi\| \\
& \leq C\left(r^{a} e^{-b r}+e^{-2(1-\kappa) r}+e^{-\eta r \sin \frac{\pi}{m}} r^{a} e^{-b r}\right. \\
& \left.\quad+e^{-\eta r \sin \frac{\pi}{m}} e^{-(2-\eta)(1-\kappa) r}\right)\left(r^{a} e^{-b r}+e^{-(1-\kappa) r}\right)\|\varphi\| \\
& \leq C e^{-(1-\tau) 2 b r}\|\varphi\|,
\end{aligned}
$$

where we choose $(2-\eta)(1-\kappa)>b, \tau>0$ sufficiently small. Thus, the result follows.

\section{Proof of the main result}

In this section we will prove Theorem 1.2.

Proof of Theorem 1.2. Let $\varphi(r)$ be the map obtained in Proposition 2.2. Define

$$
\mathcal{F}(r)=I\left(U_{\mathbf{y}}+\varphi(r)\right), \quad \forall r \in \Lambda_{m} .
$$


It is well-known that if $r$ is a critical point of $\mathcal{F}(r)$, then $U_{\mathbf{y}}+\varphi(r)$ is a solution of (1.6) (see [6]). As a consequence, in order to complete the proof of the Theorem, we only need to prove that $\mathcal{F}(r)$ has a critical point in $\Lambda_{m}$.

Hence, by Proposition 2.2 and Lemma A.3, we have

$$
\begin{aligned}
& \mathcal{F}(r)=I\left(U_{\mathbf{y}}\right)+f(\varphi)+\frac{1}{2}\langle\mathcal{L} \varphi, \varphi\rangle+R(\varphi)=I\left(U_{\mathbf{y}}\right)+O\left(\left\|f_{m}\right\|\|\varphi\|+\|\varphi\|^{2}\right) \\
& =A+\alpha B r^{2 a} e^{-2 b r}-B^{\prime} r^{-1} e^{-2 r \sin \frac{\pi}{m}}+D \alpha r^{2 a} e^{-2 b r} \sum_{j=2}^{m} V\left(y_{j}-y_{1}\right)+\alpha O\left(e^{-2 b(1+\tau) r}\right) \\
& \quad+O\left(e^{-(1+\tau) 2 r \sin \frac{\pi}{m}}\right)+O\left(\alpha e^{-(1-\tau) 2 b r}+e^{-\min \{p-1-\sigma, 2-\sigma\} r \sin \frac{\pi}{m}}\right)^{2} \\
& =A+\alpha B r^{2 a} e^{-2 b r}-B^{\prime} r^{-1} e^{-2 r \sin \frac{\pi}{m}}+D \alpha r^{2 a} e^{-2 b r} \sum_{j=2}^{m} V\left(y_{j}-y_{1}\right)+\alpha O\left(e^{-2 b(1+\tau) r}\right) \\
& \quad+O\left(e^{-(1+\tau) 2 r \sin \frac{\pi}{m}}\right),
\end{aligned}
$$

where $A, B, B^{\prime}$ and $D$ are defined in Lemma A.3. We consider its minimum respect to $r$ :

$$
\min \left\{\mathcal{F}(r): r \in \Lambda_{m}\right\}
$$

Assume that (3.1) is achieved by some $r_{m}$ in $\Lambda_{m}$, we will prove that $r_{m}$ is an interior point of $\Lambda_{m}$. Here, we only consider the case $b>\sin \frac{\pi}{m}$, using the same method, we can obtain the result in the case $b<\sin \frac{\pi}{m}$.

Investigating the following smooth function in $\Lambda_{m}$,

$$
g(r):=\alpha B r^{2 a} e^{-2 b r}-B^{\prime} r^{-1} e^{-2 r \sin \frac{\pi}{m}} .
$$

It is easy to check that $g(r)$ has a minimum point $\tilde{r}_{m}$, satisfying

$$
e^{-\left(2 b-2 \sin \frac{\pi}{m}\right) \tilde{r}_{m}}=\frac{1}{\alpha} \frac{B^{\prime}}{2 B} \frac{\tilde{r}_{m}^{-1-2 a}\left(1+2 \tilde{r}_{m} \sin \frac{\pi}{m}\right)}{b \tilde{r}_{m}-a} .
$$

Thus

$$
\tilde{r}_{m}=\left(\frac{1}{b-\sin \frac{\pi}{m}}+o(1)\right) \ln \alpha,
$$

with

$$
\begin{aligned}
g\left(\tilde{r}_{m}\right) & =\alpha B \tilde{r}_{m}^{2 a} e^{-2 b \tilde{r}_{m}}-B^{\prime} \tilde{r}_{m}^{-1} e^{-2 \tilde{r}_{m} \sin \frac{\pi}{m}} \\
& =\alpha B \tilde{r}_{m}^{2 a} e^{-2 \tilde{r}_{m} \sin \frac{\pi}{m}} \frac{1}{\alpha} \frac{B^{\prime}}{2 B} \frac{\tilde{r}_{m}^{-1-2 a}\left(1+2 \tilde{r}_{m} \sin \frac{\pi}{m}\right)}{b \tilde{r}_{m}-a}-B^{\prime} \tilde{r}_{m}^{-1} e^{-2 \tilde{r}_{m} \sin \frac{\pi}{m}} \\
& =B^{\prime} \tilde{r}_{m}^{-1} e^{-2 \tilde{r}_{m} \sin \frac{\pi}{m}}\left(\frac{\sin \frac{\pi}{m}}{b}-1+o\left(\frac{1}{\tilde{r}_{m}}\right)\right)<0 .
\end{aligned}
$$

By direct computation, we deduce that

$$
\mathcal{F}\left(r_{m}\right) \leq \mathcal{F}\left(\tilde{r}_{m}\right) \leq A+g\left(\tilde{r}_{m}\right)+O\left(\alpha^{\frac{\sin \frac{\pi}{m}+\delta}{\sin \frac{\pi}{m}-b}}\right)<A .
$$


On the other hand, we suppose that $r_{m}=\left(\frac{1+\tau}{b-\sin \frac{\pi}{m}}\right) \ln \alpha$, then

$$
\begin{aligned}
\mathcal{F}\left(r_{m}\right)= & A+\alpha B r_{m}^{2 a} e^{-2 b r_{m}}-B^{\prime} r_{m}^{-1} e^{-2 r_{m} \sin \frac{\pi}{m}}+D \alpha r_{m}^{2 a} e^{-2 b r_{m}} \sum_{j=2}^{m} V\left(y_{j}-y_{1}\right) \\
& +\alpha O\left(e^{-b(1+\tau) r_{m}}\right)+O\left(e^{-(1+\tau) 2 r_{m} \sin \frac{\pi}{m}}\right) \\
= & A+\alpha B\left(\frac{(1+\tau) \ln \alpha}{b-\sin \frac{\pi}{m}}\right)^{2 a} e^{-2 b\left(\frac{1+\tau}{b-\sin \frac{\pi}{m}}\right) \ln \alpha} \\
& -B^{\prime}\left(\frac{(1+\tau) \ln \alpha}{b-\sin \frac{\pi}{m}}\right)^{-1} e^{-2\left(\frac{1+\tau}{b-\sin \frac{\pi}{m}}\right) \ln \alpha \sin \frac{\pi}{m}} \\
& +D \alpha\left(\frac{(1+\tau) \ln \alpha}{b-\sin \frac{\pi}{m}}\right)^{2 a} e^{-2 b\left(\frac{1+\tau}{b-\sin \frac{\pi}{m}}\right) \ln \alpha} \sum_{j=2}^{m} V\left(y_{j}-y_{1}\right)+O\left(\alpha^{\frac{\sin \frac{\pi}{m}+\delta}{m}-b}\right) \\
> & A .
\end{aligned}
$$

This is a contradiction to (3.3).

Similarly

$$
\mathcal{F}\left(\left(\frac{1-\tau}{b-\sin \frac{\pi}{m}}\right) \ln \alpha\right)>A .
$$

Hence we can check that (3.1) is achieved by some $r_{m}$, which is in the interior of $\Lambda_{m}$. As a result, $r_{m}$ is a critical point of $\mathcal{F}(r)$. Therefore

$$
U_{r_{m}}+\varphi\left(r_{m}\right)
$$

is a solution of (1.6).

\section{Appendix A. Some technical estimates} Recall

In this section, we will give the energy expansion for the approximate solutions.

$$
\begin{aligned}
y_{j} & =\left(r \cos \frac{2(j-1) \pi}{m}, r \sin \frac{2(j-1) \pi}{m}, 0\right), \quad j=1, \ldots, m \\
\Omega_{j} & =\left\{x=\left(x^{\prime}, x_{3}\right) \in \mathbf{R}^{2} \times \mathbf{R}:\left\langle\frac{x^{\prime}}{\left|x^{\prime}\right|}, \frac{y_{j}^{\prime}}{\left|y_{j}^{\prime}\right|}\right\rangle \geq \cos \frac{\pi}{m}\right\}, j=1,2, \ldots, m
\end{aligned}
$$

and

$$
I(u)=\frac{1}{2} \int_{\mathbf{R}^{3}}|\nabla u|^{2}+\frac{1}{2} \int_{\mathbf{R}^{3}} u^{2}+\frac{\alpha}{4} \int_{\mathbf{R}^{3}} K(|x|) \Phi_{u} u^{2}-\frac{1}{p} \int_{\mathbf{R}^{3}}|u|^{p},
$$

where $\Phi_{u}$ is the solution of $-\Delta \Phi_{u}=K(|x|) u^{2}$.

Recall that $U$ is the unique solution of

$$
\left\{\begin{array}{l}
-\Delta u+u=u^{p-1}, \quad u>0 \text { in } \mathbf{R}^{3}, \\
u(0)=\max _{x \in \mathbf{R}^{3}} u(x) .
\end{array}\right.
$$

Let $V$ be the solution of

$$
\left\{\begin{array}{l}
-\Delta v=U^{2}, \quad \text { in } \mathbf{R}^{3} \\
v \in D^{1,2}\left(\mathbf{R}^{3}\right)
\end{array}\right.
$$


Then, $V$ is radial, and $r V(r) \rightarrow V_{0}>0$, as $r \rightarrow+\infty$.

Now, we give the following Lemma:

Lemma A.1. [2, Lemma 3.7] Given $u, u^{\prime}: \mathbf{R}^{3} \rightarrow \mathbf{R}$ two positive continuous radial functions such that:

$$
u(x) \sim|x|^{a} e^{-b|x|}, u^{\prime}(x) \sim|x|^{a^{\prime}} e^{-b^{\prime}|x|}(x \rightarrow \infty)
$$

where $a, a^{\prime} \in \mathbf{R}, b>0, b^{\prime}>0$. Let $\xi \in \mathbf{R}^{3}$ tend to infinity. Then, the following asymptotic estimates hold:

(1) If $b<b^{\prime}$,

$$
\int_{\mathbf{R}^{3}} u_{\xi} u^{\prime} \sim|\xi|^{a} e^{-b|\xi|}
$$

(2) If $b=b^{\prime}$, suppose, for simplicity, that $a \geq a^{\prime}$, then

$$
\int_{\mathbf{R}^{3}} u_{\xi} u^{\prime} \sim \begin{cases}|\xi|^{a+a^{\prime}+2} e^{-b|\xi|}, & a^{\prime}>-2 \\ |\xi|^{a} e^{-b|\xi|} \log |\xi|, & a^{\prime}=-2 \\ |\xi|^{a} e^{-b|\xi|}, & a^{\prime}<-2\end{cases}
$$

Lemma A.2. For a suitable $\sigma>0$, we have

$$
\Phi_{U_{\mathbf{y}}}(y)=r^{a} e^{-b r} \sum_{j=1}^{m} V\left(y-y_{j}\right)+O\left(\sum_{j=1}^{m} r^{a} e^{-(b+\sigma) r} \frac{1}{1+\left|y-y_{j}\right|}\right) .
$$

Proof. Firstly,

$$
U_{\mathbf{y}}^{2}=U_{y_{1}}^{2}+O\left(U_{y_{1}} \sum_{j=2}^{m} U_{y_{j}}+\left(\sum_{j=2}^{m} U_{y_{j}}\right)^{2}\right)
$$

For any $x \in \Omega_{1}$, we have

$$
\left|x-y_{i}\right| \geq \frac{1}{2}\left|y_{i}-y_{1}\right|
$$

and for any $\beta>0$,

$$
\sum_{j=2}^{m} U_{y_{j}}^{\beta}(x) \leq C \sum_{j=2}^{m} e^{-\beta\left|x-y_{j}\right|} \leq C \sum_{j=2}^{m} e^{-\frac{\beta}{2}\left|y_{1}-y_{j}\right|} \leq C e^{-\beta \frac{\pi r}{m}}, \quad x \in \Omega_{1} .
$$

As a result,

$$
U_{y_{1}} \sum_{j=2}^{m} U_{y_{j}} \leq U_{y_{1}}^{\frac{3}{2}} \sum_{j=2}^{m} U_{y_{j}}^{\frac{1}{2}} \leq C e^{-\frac{1}{2}\left|x-y_{1}\right|} e^{-\frac{\pi r}{2 m}}, \quad x \in \Omega_{1},
$$

and

$$
\left(\sum_{j=2}^{m} U_{y_{j}}\right)^{2} \leq U_{y_{1}}\left(\sum_{j=2}^{m} U_{y_{j}}^{\frac{1}{2}}\right)^{2} \leq C e^{-\frac{1}{2}\left|x-y_{1}\right|} e^{-\frac{\pi r}{m}}, \quad x \in \Omega_{1} .
$$

So,

$$
U_{\mathbf{y}}^{2}=U_{y_{1}}^{2}+O\left(e^{-\frac{1}{2}\left|x-y_{1}\right|} e^{-\frac{\pi r}{2 m}}\right)
$$


By Lemma A.1 and the above result, we are led to

$$
\begin{aligned}
& \int_{\Omega_{j}} \frac{K(|y|)}{|x-y|} U_{\mathbf{y}}^{2}(y) d y=\int_{\Omega_{j}} \frac{K(|y|)}{|x-y|}\left(U_{y_{j}}^{2}+O\left(e^{-\frac{1}{2}\left|y-y_{j}\right|} e^{-\frac{\pi r}{2 m}}\right)\right) d y \\
& =\int_{\Omega_{j} \cap B_{\frac{r}{2}}(0)} \frac{K\left(\left|y+y_{j}\right|\right)}{\left|x-y-y_{j}\right|} U^{2} d y+O\left(\int_{\mathbf{R}^{3} \backslash B_{\frac{r}{2}}(0)} \frac{K\left(\left|y+y_{j}\right|\right)}{\left|x-y-y_{j}\right|} U^{2} d y\right) \\
& \quad+O\left(r^{a} e^{-(b+\sigma) r} \frac{1}{1+\left|y-y_{j}\right|}\right) \\
& =\int_{\Omega_{j} \cap B_{\frac{r}{2}}(0)}(1+o(1))\left|y+y_{j}\right|^{a} e^{-b\left|y+y_{j}\right|} \frac{U^{2}}{\left|x-y-y_{j}\right|} d y \\
& +O\left(\int_{\mathbf{R}^{3} \backslash B_{\frac{r}{2}}(0)} \frac{K\left(\left|y+y_{j}\right|\right)}{\left|x-y-y_{j}\right|} U^{2} d y\right)+O\left(r^{a} e^{-(b+\sigma) r} \frac{1}{1+\left|y-y_{j}\right|}\right) \\
& =r^{a} e^{-b r} V\left(y-y_{j}\right)+O\left(r^{a} e^{-(b+\sigma) r} \frac{1}{1+\left|y-y_{j}\right|}\right) .
\end{aligned}
$$

So

$$
\Phi_{U_{\mathbf{y}}}(y)=r^{a} e^{-b r} \sum_{j=1}^{m} V\left(y-y_{j}\right)+O\left(\sum_{j=1}^{m} r^{a} e^{-(b+\sigma) r} \frac{1}{1+\left|y-y_{j}\right|}\right) .
$$

Lemma A.3. We have

$$
\begin{aligned}
I\left(U_{r}\right)= & A+\alpha B r^{2 a} e^{-2 b r}-B^{\prime} r^{-1} e^{-2 r \sin \frac{\pi}{m}}+D \alpha r^{2 a} e^{-2 b r} \sum_{j=2}^{m} V\left(y_{j}-y_{1}\right) \\
& +\alpha O\left(e^{-2 b(1+\tau) r}\right)+O\left(e^{-(1+\tau) 2 r \sin \frac{\pi}{m}}\right),
\end{aligned}
$$

where $A=m\left(\frac{1}{2}-\frac{1}{p}\right) \int_{\mathbf{R}^{3}} U^{p}, B=\frac{m}{4} \int_{\mathbf{R}^{3}} V U^{2}$ and $D=\frac{m}{4} \int_{\mathbf{R}^{3}} U^{2}, \tau>0$ sufficiently small.

Proof. Recall that

$$
I\left(U_{\mathbf{y}}\right)=\frac{1}{2} \int_{\mathbf{R}^{3}}\left|\nabla U_{\mathbf{y}}\right|^{2}+\frac{1}{2} \int_{\mathbf{R}^{3}} U_{\mathbf{y}}^{2}+\frac{\alpha}{4} \int_{\mathbf{R}^{3}} K(|x|) \Phi_{U_{\mathbf{y}}} U_{\mathbf{y}}^{2}-\frac{1}{p} \int_{\mathbf{R}^{3}}\left|U_{\mathbf{y}}\right|^{p},
$$

By direct computation, we have

$$
\frac{1}{2} \int_{\mathbf{R}^{3}}\left|\nabla U_{\mathbf{y}}\right|^{2}+\frac{1}{2} \int_{\mathbf{R}^{3}} U_{\mathbf{y}}^{2}=\frac{m}{2} \int_{\mathbf{R}^{3}} U^{p}+\frac{m}{2} \sum_{j=2}^{m} \int_{\mathbf{R}^{3}} U_{y_{1}}^{p-1} U_{y_{j}} .
$$

We also have

$$
\begin{aligned}
\int_{\mathbf{R}^{3}}\left|U_{\mathbf{y}}\right|^{p}= & m \int_{\mathbf{R}^{3}} U_{y_{1}}^{p}+m p \int_{\mathbf{R}^{3}} \sum_{k=2}^{m} U_{y_{1}}^{p-1} U_{y_{k}} \\
& +\left\{\begin{array}{l}
O\left(\sum_{k \neq j} \int_{\mathbf{R}^{3}} U_{y_{k}}^{\frac{p}{2}} U_{y_{j}}^{\frac{p}{2}}\right), \quad \text { if } 2<p \leq 3, \\
O\left(\sum_{k \neq j} \int_{\mathbf{R}^{3}} U_{y_{k}}^{p-2} U_{y_{j}}^{2}\right), \quad \text { if } p>3, \\
=
\end{array}\right. \\
& \int_{\mathbf{R}^{3}} U^{p}+m p \int_{\mathbf{R}^{3}}\left|U_{y_{1}}\right|^{p-1} \sum_{j=2}^{m} U_{y_{j}}+m O\left(e^{-(1+\tau)\left|y_{2}-y_{1}\right|}\right),
\end{aligned}
$$


where $\tau>0$ sufficiently small.

Using Lemma A.2, we see

$$
\begin{aligned}
& \int_{\mathbf{R}^{3}} K(|x|) \Phi_{U_{\mathbf{y}}} U_{\mathbf{y}}^{2}=m \int_{\Omega_{1}} K(|x|) \Phi_{U_{\mathbf{y}}} U_{\mathbf{y}}^{2} \\
& =m \int_{\Omega_{1}} K(|x|)\left(r^{a} e^{-b r} \sum_{j=1}^{m} V\left(y-y_{j}\right)\right. \\
& \left.+O\left(\sum_{j=1}^{m} r^{a} e^{-(b+\sigma) r} \frac{1}{1+\left|y-y_{j}\right|}\right)\right)\left(U_{y_{1}}^{2}+O\left(e^{-\frac{1}{2}\left|x-y_{1}\right|} e^{-\frac{\pi r}{2 m}}\right)\right) \\
& =m \int_{\Omega_{1}} K(|x|) r^{a} e^{-b r} \sum_{j=1}^{m} V\left(y-y_{j}\right) U_{y_{1}}^{2} \\
& +m \int_{\Omega_{1}} K(|x|) r^{a} e^{-b r} \sum_{j=1}^{m} V\left(y-y_{j}\right) O\left(e^{-\frac{1}{2}\left|x-y_{1}\right|} e^{-\frac{\pi r}{2 m}}\right) \\
& +m \int_{\Omega_{1}} K(|x|) O\left(\sum_{j=1}^{m} r^{a} e^{-(b+\sigma) r} \frac{1}{1+\left|y-y_{j}\right|}\right) U_{y_{1}}^{2} \\
& +m \int_{\Omega_{1}} K(|x|) O\left(\sum_{j=1}^{m} r^{a} e^{-(b+\sigma) r} \frac{1}{1+\left|y-y_{j}\right|}\right) O\left(e^{-\frac{1}{2}\left|x-y_{1}\right|} e^{-\frac{\pi r}{2 m}}\right) \\
& =m \int_{\Omega_{1}} K(|x|) r^{a} e^{-b r} \sum_{j=1}^{m} V\left(y-y_{j}\right) U_{y_{1}}^{2} \\
& +m \int_{\Omega_{1}} K(|x|) O\left(\sum_{j=1}^{m} r^{a} e^{-(b+\sigma) r} \frac{1}{1+\left|y-y_{j}\right|}\right) U_{y_{1}}^{2} \\
& +O\left(k r^{a} e^{-b(1+\delta) r} \int_{\mathbf{R}^{3}} K(|x|) \sum_{j=1}^{m} \frac{1}{1+\left|y-y_{j}\right|} e^{-\frac{1}{2}\left|x-y_{1}\right|}\right) \\
& =m r^{2 a} e^{-2 b r} \int_{\mathbf{R}^{3}} V U^{2}+m r^{2 a} e^{-2 b r} \sum_{j=2}^{m} V\left(y_{j}-y_{1}\right) \int_{\mathbf{R}^{3}} U^{2} \\
& +m O\left(r^{2 a} e^{-2 b(1+\delta) r} \sum_{j=2}^{m} \frac{1}{\left|y_{1}-y_{j}\right|}\right)+m O\left(r^{2 a} e^{-2 b(1+\delta) r}\right),
\end{aligned}
$$

where $\delta>0$ is a suitable constant.

Above all, we deduce

$$
\begin{aligned}
I\left(U_{\mathbf{y}}\right)= & \frac{1}{2} \int_{\mathbf{R}^{3}}\left|\nabla U_{\mathbf{y}}\right|^{2}+\frac{1}{2} \int_{\mathbf{R}^{3}} U_{\mathbf{y}}^{2}+\frac{\alpha}{4} \int_{\mathbf{R}^{3}} K(|x|) \Phi_{U_{\mathbf{y}}} U_{\mathbf{y}}^{2}-\frac{1}{p} \int_{\mathbf{R}^{3}}\left|U_{\mathbf{y}}\right|^{p} \\
= & \frac{m}{2} \int_{\mathbf{R}^{3}} U^{p}+\frac{m}{2} \sum_{j=2}^{m} \int_{\mathbf{R}^{3}} U_{y_{1}}^{p-1} U_{y_{j}}-\frac{1}{p} m \int_{\mathbf{R}^{3}} U^{p}-m \int_{\mathbf{R}^{3}} U_{y_{1}}^{p-1} \sum_{j=2}^{m} U_{y_{j}} \\
& +m O\left(e^{-(1+\tau)\left|y_{2}-y_{1}\right|}\right) \\
& +\frac{\alpha}{4} m r^{2 a} e^{-2 b r} \int_{\mathbf{R}^{3}} V U^{2}+\frac{\alpha}{4} m r^{2 a} e^{-2 b r} \sum_{j=2}^{m} V\left(y_{j}-y_{1}\right) \int_{\mathbf{R}^{3}} U^{2}
\end{aligned}
$$




$$
\begin{aligned}
& +m \alpha O\left(r^{2 a} e^{-2 b(1+\delta) r} \sum_{j=2}^{m} \frac{1}{\left|y_{1}-y_{j}\right|}\right)+m \alpha O\left(r^{2 a} e^{-2 b(1+\delta) r}\right) \\
= & m\left(\frac{1}{2}-\frac{1}{p}\right) \int_{\mathbf{R}^{3}} U^{p}-\frac{m}{2} \int_{\mathbf{R}^{3}}\left(U_{y_{1}}^{p-1} U_{y_{2}}+U_{y_{1}}^{p-1} U_{y_{m}}\right) \\
& +\frac{\alpha}{4} m r^{2 a} e^{-2 b r} \int_{\mathbf{R}^{3}} V U^{2} \\
& +\frac{\alpha}{4} m r^{2 a} e^{-2 b r} \sum_{j=2}^{m} V\left(y_{j}-y_{1}\right) \int_{\mathbf{R}^{3}} U^{2}+m O\left(e^{-(1+\tau)\left|y_{2}-y_{1}\right|}\right) \\
& +O\left(r^{2 a} e^{-2 b(1+\delta) r} \sum_{j=2}^{m} \frac{1}{\left|y_{1}-y_{j}\right|}\right)+m \alpha O\left(r^{2 a} e^{-2 b(1+\delta) r}\right) \\
= & m\left(\frac{1}{2}-\frac{1}{p}\right) \int_{\mathbf{R}^{3}} U^{p}+\frac{\alpha}{4} m r^{2 a} e^{-2 b r} \int_{\mathbf{R}^{3}} V U^{2} \\
& +\frac{\alpha}{4} m r^{2 a} e^{-2 b r} \sum_{j=2}^{m} V\left(y_{j}-y_{1}\right) \int_{\mathbf{R}^{3}} U^{2} \\
& -B^{\prime} r^{-1} e^{-2 r \sin \frac{\pi}{m}}+\alpha O\left(e^{-2 b(1+\tau) r}\right)+O\left(e^{-(1+\tau) 2 r \sin \frac{\pi}{m}}\right),
\end{aligned}
$$

where $\tau>0$ sufficiengly small.

\section{References}

[1] Alves, C. O., and M. A. Souto: Existence of least energy nodal solution for a SchrödingerPoisson system in bounded domains. - Z. Angew. Math. Phys. 65, 2014, 1153-1166.

[2] Ambrosetti, A., E. Colorado, and D. Ruiz: Multi-bump solitons to linearly coupled systems of nonlinear Schrödinger equations. - Calc. Var. Partial Differential Equations 30, 2007, 85-112.

[3] Ambrosetti, A., and D. Ruiz: Multiple bound states for the Schrödinger-Poisson problem. - Comm. Contemp. Math. 10, 2008, 392-404.

[4] Azzollini, A., and A. Pomponio: Ground state solutions for the nonlinear SchrödingerMaxwell equations. - J. Math. Anal. Appl. 345, 2008, 90-108.

[5] Benci, V., and D. Fortunato: An eigenvalue problem for the Schrödinger-Maxwell equations. - Topol. Methods Nonlinear Anal. 11, 1998, 283-293.

[6] CAO, D., and Z. TANG: Existence and Uniqueness of multi-bump bound states of nonlinear Schrödinger equations with electromagnetic fields. - J. Differential Equations 222, 2006, 381424.

[7] Chen, S., and C. WANG: Existence of multiple nontrivial solutions for a Schrödinger-Poisson system. - J. Math. Anal. Appl. 411, 2014, 787-793.

[8] DANCER, E. N., and S. YAn: On positive multipeak solutions of a nonlinear elliptic problem. - J. Lond. Math. Soc. 62, 2000, 213-227.

[9] D'Aprile, T., and D. Mugnai: Solitary waves for nonlinear Klein-Gordon-Maxwell and Schrödinger-Maxwell equations. - Proc. Roy. Soc. Edinburgh Sect. A 134, 2004, 893-906.

[10] He, X., and W. Zou: Existence and concentration of ground states for Schrödinger-Poisson equations with critical growth. - J. Math. Phys. 53, 2012, 023702, 1-20.

[11] HE, Y., and G. LI: Standing waves for a class of Schrödinger-Poisson equations in $\mathbf{R}^{3}$ involving critical sobolev exponents. - Ann. Acad. Sci. Fenn. Math. 40, 2015, 729-766. 
[12] IAnNi, I.: Solutions of the Schrödinger-Poisson problem concentrating on spheres. Part II: Existence. - Math. Models Meth. Appl. Sci. 19, 2009, 877-910.

[13] Ianni, I., and G. Vaira: On concentration of positive bound states for the SchrödingerPoisson problem with potentials. - Adv. Nonlinear Stud. 8, 2008, 573-595.

[14] Ianni, I., and G. Vaira: Solutions of the Schrödinger-Poisson problem concentrating on spheres. Part I: Necessary conditions. - Math. Models Meth. Appl. Sci. 19, 2009, 707-720.

[15] KIM, S., and J. SEOK: On nodal solutions of the nonlinear Schrödinger-Poisson equations. Comm. Contemp. Math. 14, 2012, 1-16.

[16] Kwong, M. K.: Uniqueness of the positive solution of $\Delta u-u+u^{p}=0$ in $\mathbf{R}^{n}$. - Arch. Ration. Mech. Anal. 105, 1989, 243-266.

[17] LI, G., S. PENG, and S. YAn: Infinitely many positive solutions for the nonlinear SchrödingerPoisson system. - Commun. Contemp. Math. 6, 2010, 1069-1092.

[18] Lin, L., Z. LiU, and S. Chen: Multi-bump solutions for a semilinear Schrödinger equation. Indiana Univ. Math. J. 58, 2009, 1659-1689.

[19] Long, W., and S. Peng: Multiple positive solutions for a type of nonlinear Schrödinger equations. - Ann. Sc. Norm. Super. Pisa Cl. Sci. (5) 16, 2016, 603-623.

[20] Ruiz, D.: The Schrödinger-Poisson equation under the effect of a nonlinear local term. - J. Funct. Anal. 237, 2006, 655-674.

[21] Ruiz, D., and G. VAira: Cluster solutions for the Schrödinger-Poisson-Slater problem around a local minimum of potential. - Rev. Mat. Iberoam. 27, 2011, 253-271.

[22] Sun, J., T. Wu, and Y. Wu: On the Schrödinger-Poisson system with steep potential well and indefinite potential. - arXiv:1412.5463v1.

[23] Wang, Z., and H. Zhou: Sign-changing solutions for the nonlinear Schrödinger-Poisson system in $\mathbf{R}^{3}$. - Calc. Var. Partial Differential Equations 52, 2015, 927-943.

[24] Zhang, J.: The existence and concentration of positive solutions for a nonlinear SchrödingerPoisson system with critical growth. - J. Math. Phys. 55, 2014, 031507, 1-15.

[25] Zhao, L., H. LiU, and F. ZhaO: Existence and concentration of solutions for the SchrödingerPoisson equations with steep well potential. - J. Differential Equations 255, 2013, 1-23.

Received 29 January 2016 • Accepted 5 August 2016 